\title{
PREVALENCIA DE SOBREPESO Y OBESIDAD EN NIÑOS MENORES DE CINCO AÑOS EN EL PERÚ 2007-2010
}

\author{
Jaime Pajuelo-Ramírez ${ }^{1,2, a, b}$, Marianella Miranda-Cuadros, 3 ,c, Miguel Campos-Sánchez ${ }^{4, a, d}$, \\ José Sánchez-Abanto $3, b, c$
}

\begin{abstract}
RESUMEN
Objetivos. Estimar la prevalencia de sobrepeso y obesidad en niños menores de cinco años en el Perú durante los años 2007 a 2010 y describir su distribución de acuerdo con ámbitos geográficos, niveles de pobreza, edad, educación de la madre, lactancia materna exclusiva, sexo y peso al nacer. Materiales y métodos: Se realizó una encuesta continua (transversal repetida), por muestreo aleatorio multietápico, del universo de niños menores de cinco años y gestantes residentes en el Perú, dividido en cinco ámbitos geográficos. Las mediciones antropométricas se efectuaron de acuerdo con metodología estándar internacional. Resultados: Se estudiaron 3669 niños menores de cinco años, de ellos 50,3\% fueron niños. En Lima Metropolitana residían 680; 763 en el resto de costa; 719 en la sierra urbana, 699 en la sierra rural y 808 en la selva. La prevalencia nacional de sobrepeso y obesidad es $6,9 \%$, con la mayor prevalencia, en Lima Metropolitana $(10,1 \%)$ y con menor prevalencia en la selva $(2,6 \%)$. Mediante regresión logística múltiple se identificaron como factores asociados a la edad, el sexo, el ámbito geográfico y el peso al nacer. Conclusiones: Los factores asociados con sobrepeso y obesidad son la procedencia de Lima Metropolitana, el primer año de vida, el sexo masculino y el peso al nacer mayor a $2,5 \mathrm{~kg}$.
\end{abstract}

Palabras clave: Obesidad; Sobrepeso; Preescolar; Lactante; Encuestas nutricionales; Perú (fuente: DeCS BIREME).

\section{PREVALENCE OF OVERWIGHT AND OBESITY AMONG CHILDREN UNDER FIVE YEARS IN PERU 2007-2010}

\begin{abstract}
Objectives: To estimate the prevalence of overweight and obesity in children under five in Peru in the years 2007-2010 and to describe according to geographical areas, poverty levels, maternal education, breastfeeding, child age, sex and birth weight. Materials and methods: continuous (repeated cross-sectional) multistage, random sampling survey from the universe of children under five-years and pregnant women living in Peru, divided into five geographical areas. $R \mathbf{e}-$ sults: Out of 3,669 children, $50.3 \%$ were males (Lima $N=680$, Remaining Coast $N=763$, Urban Sierra $N=719$, Rural Sierra $N=699$, Jungle $N=808$ ) having their weight and height measured according to international standards. The national prevalence of overweight and obesity was $6.9 \%$, with Metropolitan Lima $(10.1 \%)$ as the highest and in the Jungle $(2.6 \%)$ as the lowest. Age, sex, geographical area and birth weight were identified as risk factors through multiple logistic regression. Conclusions: overweight and obesity are higher in Lima, during the first year of age and when birth weight is more than $2.5 \mathrm{Kg}$.
\end{abstract}

Key words: Obesity; Overweight; Infant; Child, preschool; Infant; Nutrition surverys; (source: MeSH NLM).

\section{INTRODUCCIÓN}

Desde hace más de diez años, la Organización Mundial de la Salud (OMS) ha declarado a la obesidad como la epidemia del siglo XXI ${ }^{(1)}$, condición cuya relevancia no solo se centra en el incremento constante de su incidencia, sino que trasciende hacia el aumento de las morbilidades asociadas - como diabetes mellitus tipo 2 (DM-2), dislipidemias, la hipertensión arterial (2) y cierto tipo de cánceres ${ }^{(3)}$ - que comprometen muy seriamente la salud a temprana edad ${ }^{(4)}$.
Se estima que en el mundo hay 22 millones de niños menores de cinco años con obesidad, asimismo, se ha reportado un incremento de la prevalencia del sobrepeso y la obesidad en todos los grupos de edad en Estados Unidos de América (EUA) durante la última década, el cual es más marcado en escolares y adolescentes, grupos de edad donde se ha duplicado y triplicado, respectivamente, desde $1980^{(5,6)}$. Sin embargo, en Europa ${ }^{(7)}$ y América Latina ${ }^{(8)}$ se observa un incremento paulatino y sostenido de su incidencia, condición que se ha relacionado con cambios en los

\footnotetext{
Instituto de Investigaciones Clínicas, Facultad de Medicina, Universidad Nacional Mayor de San Marcos. Lima, Perú.

Servicio de Endocrinología, Hospital Nacional 2 de Mayo. Lima, Perú.

Dirección Ejecutiva de Vigilancia Alimentaria y Nutricional, Centro Nacional de Alimentación y Nutrición, Instituto Nacional de Salud. Lima, Perú.

Departamento de Ciencias Exactas, Facultad de Ciencias y Filosofía, Universidad Peruana Cayetano Heredia. Lima, Perú.

Médico; ${ }^{b}$ Magíster en Nutricion; c Nutricionista; ${ }^{d}$ Doctor of Phylosophy
}

Recibido: 28-09-10 Aprobado: 27-04-11 
estilos de vida, fundamentalmente con el aumento en la ingesta de energía y disminución de la actividad diaria ${ }^{(9)}$.

En Perú han existido varias aproximaciones para cuantificar el problema; la Encuesta Nacional de Nutrición y Salud (ENNSA) fue la primera que reportó que un $4 \%$ de niños menores de cinco años presentaron el indicador Peso para laTalla $(P T)>2 z{ }^{(10)}$. Posteriormente el Instituto Nacional de Estadística e Informática (INEI), en sus Encuestas Demográfica y de Salud Familiar (ENDES) encontró una prevalencia de PT $>2 z$ del 5,1\%, 5,5\% y 6,5\% para los años 1992 , 1996 y 2000; respectivamente ${ }^{(11)}$. El Centro Nacional de Alimentación y Nutrición (CENAN), por intermedio del Monitoreo Nacional de Indicadores Nutricionales (MONIN) informó que la prevalencia de $\mathrm{PT}>2 \mathrm{z}$ en el año 2003 fue de $5,4 \%{ }^{(12)}$ y en el 2004 de $5,7 \%{ }^{(13)}$.

Asimismo, en Perú también se ha reportado la presencia de complicaciones metabólicas en niños mayores y adolescentes, como resistencia a la insulina (24\%) ${ }^{(14)}$, dislipidemias mixtas (26\%), hipercolesterolemia (33\%) e hipertrigliceridemia (56\%), factores que conducen a la ateroesclerosis ${ }^{(15)}$ y que junto con la obesidad conforman el síndrome metabólico $(8,8 \%)$ un marcador directo de riesgo cardiovascular (16); es en este contexto que un abordaje de estas condiciones en lactantes y preescolares es relevante.

Se ha demostrado que el proceso de aterosclerosis se inicia en la niñez y es progresivo, además, se ha observado una fuerte asociación entre la obesidad y la presencia incrementada de lesiones ateroscleróticas tanto en la arteria aorta como en las arterias coronarias ${ }^{(4)}$. También se ha observado que el incremento del peso durante la niñez es un fuerte predictor de calcificación coronaria en el futuro ${ }^{(17)}$. Asimismo, se han encontrado una mayor frecuencia de lesiones de grosor de la intima de la carótida y disfunción endotelial en niños obesos en relación a los que tienen un peso adecuado ${ }^{(18)}$.

Otro factor por considerar, y no menos importante que los expuestos previamente, son las consecuencias dentro de la esfera psicosocial que se hacen más dramáticas cuando se llega a la adolescencia; la baja autoestima, el pobre desempeño social, la discriminación y las pocas oportunidades para superarse ${ }^{(19)}$.

El objetivo del presente estudio fue estimar la prevalencia de sobrepeso y obesidad $(P T>2 z)$ en niños menores de cinco años y describir su distribución según ámbitos geográficos, niveles de pobreza, edad, educación de la madre, lactancia materna exclusiva, sexo y peso al nacer.

\section{MATERIALES Y MÉTODOS}

\author{
TIPO DE ESTUDIO, POBLACIÓN Y MUESTREO
}

Se desarrolló un análisis secundario de datos del Monitoreo Nacional de Indicadores Nutricionales (MONIN), encuesta nutricional de base poblacional llevada a cabo por el CENAN durante los años 2007 a $2010^{(20,21)}$ con el objetivo principal de medir la frecuencia de la desnutrición infantil y sus factores asociados (incluyendo las intervenciones). EI MONIN es una encuesta continua (transversal repetida) con un muestreo aleatorio multietápico teniendo como universo los niños menores de cinco años y las mujeres gestantes residentes en el Perú. Considera cinco ámbitos geográficos y seis periodos aproximadamente trimestrales (semanas permutadas aleatoriamente que fueron $78 \%$ del tiempo entre el 19 noviembre de 2007 y el 02 de abril de 2010).

\section{CÁLCULO DEL TAMAÑO DE LA MUESTRA}

El cálculo del tamaño de muestra del estudio principal fue realizado con un $95 \%$ de confianza, un margen de error de 7,5-15\%, una prevalencia estimada entre 20 a $80 \%$ (efecto de diseño de 1,5 ) resultando en un total deseado de 3600 individuos; el alcanzado fue de 3827 . En el caso de este estudio en particular, se consideró solamente a los niños menores de cinco años que tuvieron puntaje $z$ de peso y talla calculados en la base de datos publicada por CENAN (http://www.ins.gob.pe/ insvirtual/BiblioDig); de los cuales 1847 fueron varones y 1822 mujeres. Residían en Lima Metropolitana 680, en el resto de costa 763, en la sierra urbana 719, en la sierra rural 699 y en la selva 808.

\section{VARIABLES Y MEDICIONES}

Las mediciones antropométricas fueron realizadas de acuerdo con metodología internacionalmente aceptada, incluida en la guía elaborada por CENAN (22), utilizándose balanzas digitales calibradas con una precisión de $\pm 100 \mathrm{~g}$ y tallímetros confeccionados de acuerdo con las especificaciones técnicas de este mismo centro ${ }^{(23)}$. Para fines del diagnóstico se utilizó la población de referencia de la Organización Mundial de la Salud (OMS 2006) ${ }^{(23)}$ y se definió como niños con sobrepeso u obesidad a aquellos cuya razón peso/talla se encontrase en dos o más desviaciones estándar de la referencia ${ }^{(24)}$. El peso al nacer se obtuvo del carné de crecimiento y desarrollo (CRED).

Para medir el nivel de pobreza, se consideró los quintiles definidos por el Fondo de Cooperación para el Desarrollo Social (FONCODES), los cuales se basan en la presencia 


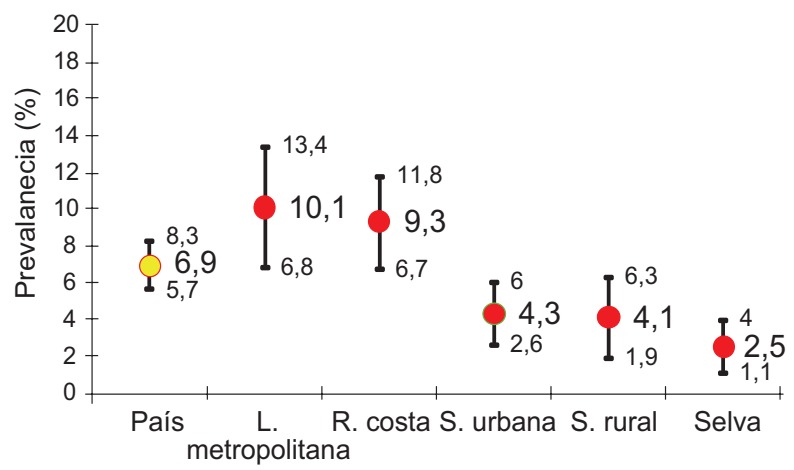

Figura 1. Prevalencia de sobrepeso y obesidad en niños menores de cinco años, nacional y según ámbitos, MONIN Perú, noviembre 2007 a marzo 2010.

de carencia o necesidad básicas insatisfechas (agua, desagüe y electricidad) y de vulnerabilidad (analfabetismo, proporción de niños de 0 a 12 años y desnutrición crónica en niños de 6 a 9 años), siendo el primer quintil el más pobre y el quinto el menos pobre ${ }^{(25)}$

La educación fue evaluada en base a lo que la madre declaró. En ese sentido, se consideró analfabetas aquellas que declararon no saber leer ni escribir; con educación primaria aquellas con educación preescolar y primaria, secundaria a las que terminaron la secundaria y superior a los profesionales y técnicos.

\section{ANÁLISIS ESTADÍSTICO}

Para evaluar la asociación entre el sobrepeso y obesidad con los factores estudiados, se aplicó regresión logística ajustada al diseño muestral en la siguiente secuencia: primero se aplicaron regresiones simples con cada factor como única variable independiente, luego se aplicó una regresión múltiple con todos los factores estudiados, de la cual se fueron retirando uno a uno los factores empezando por el menos significativo y finalmente se aplicó una regresión logística final a los factores remanentes.

Los datos fueron analizados mediante el paquete estadístico SPSS versión 15.0 con módulo Complex Samples ${ }^{(26)}$.

\section{RESULTADOS}

\section{PREVALENCIA DE OBESIDAD Y SOBREPESO}

En La Figura 1 se presenta la prevalencia encontrada y sus intervalos de confianza corregidos por el tipo de muestreo al $95 \%$, en todos los estratos estudiados. Existe un $6,9 \%$ de niños con sobrepeso y obesidad

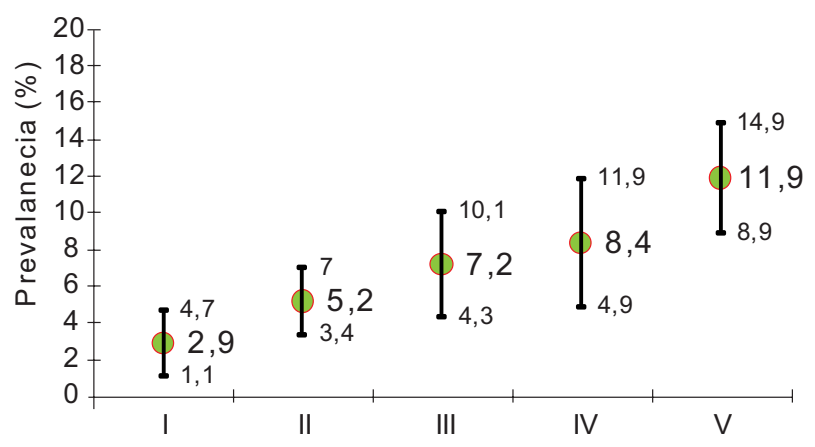

Figura 2. Prevalencia de sobrepeso y obesidad en niños menores de cinco años, según quintiles de pobreza, MONIN Perú, noviembre 2007 a marzo 2010.

$(\mathrm{PT}>2 \mathrm{z})$ en todo el país. Superan esta prevalencia en Lima Metropolitana $(10,1 \%)$ y resto de costa $(9,3 \%)$ y por debajo de la misma la sierra urbana $(4,3 \%)$ y rural $(4,1 \%)$, lo mismo que la selva $(2,5 \%)$.

\section{FACTORES ASOCIADOS Y ANÁLISIS ESTADÍSTICO POR ETAPAS}

La prevalencia de sobrepeso y obesidad parece aumentar conforme disminuyen los indicadores de pobreza (Figura 2), asimismo, en lo referente a la edad, parece existir mayor prevalencia en los grupos extremos (Figura 3).

En términos generales, un $8,5 \%$ de niños con peso al nacer $>2500 \mathrm{~kg}$ y un $3,7 \%$ sin lactancia materna exclusiva, tienen sobrepeso y obesidad.

La Tabla 1 muestra los resultados de significancia para cada uno de los factores en las tres etapas del análisis. Se encuentra clara asociación de la prevalencia de sobrepeso con el estrato geográfico, la edad, el sexo y el peso al nacer.

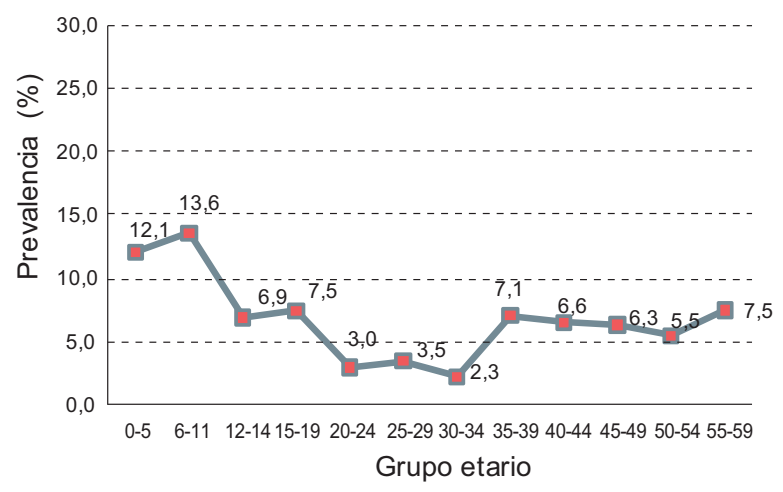

Figura 3. Prevalencia de sobrepeso y obesidad de niños menores de cinco años, según grupos de edad, MONIN Perú, noviembre 2007 a marzo 2010. 
Tabla 1. Análisis por etapas de los factores asociados a sobrepeso y obesidad en niños menores de cinco años. MONIN Perú, noviembre 2007 a marzo 2010.

\begin{tabular}{|c|c|c|c|c|c|c|c|}
\hline Variable o Término & N1 & P1 & P2 & P3 & B3 & S3 & O3 (LI y LS 95\%) \\
\hline Intercepto & & & 0,00 & 0,00 & $-4,34$ & 0,81 & \\
\hline Estrato & 3669 & 0,00 & 0,06 & 0,00 & & & \\
\hline Estrato - Costa & & & 0,76 & 0,86 & $-0,04$ & 0,24 & $0,96(0,60-1,54)$ \\
\hline Estrato - Sierra Urbana & & & 0,11 & 0,01 & $-0,71$ & 0,28 & $0,49(0,28-0,86)$ \\
\hline Estrato - Sierra Rural & & & 0,79 & 0,15 & $-0,54$ & 0,38 & $0,58(0,28-1,22)$ \\
\hline Estrato - Selva & & & 0,05 & 0,00 & $-1,30$ & 0,37 & $0,27(0,13-0,56)$ \\
\hline \multicolumn{8}{|l|}{ Estrato - Lima Metropolina } \\
\hline Educación Materna & 3587 & 0,00 & 0,16 & & & & \\
\hline Educación Materna - Ninguna & & & 0,71 & & & & \\
\hline Educación Materna - Primaria & & & 0,49 & & & & \\
\hline Educación Materna - Secundaria & & & 0,19 & & & & \\
\hline \multicolumn{8}{|l|}{ Educación Materna - Superior } \\
\hline Edad (m) & 3669 & 0,00 & 1,00 & & & & \\
\hline Edad (m) - Log, Neperiano & & & 0,12 & 0,00 & $-0,30$ & 0,08 & $0,74(0,64-0,86)$ \\
\hline Sexo $(1 \mathrm{M}, 0 \mathrm{~F})$ & 3669 & 0,00 & 0,01 & 0,02 & 0,55 & 0,23 & $1,74(1,11-2,71)$ \\
\hline Quintil FonCoDes 2006 & 3656 & 0,00 & 0,28 & & & & \\
\hline Lactancia Exclusiva (1 sí, 0 no) & 3666 & 0,03 & 0,67 & & & & \\
\hline Peso al Nacer (kg) & 2987 & 0,00 & 0,00 & 0,00 & 0,79 & 0,24 & $2,20(1,39-3,50)$ \\
\hline NX & & & 2,922 & 2,987 & & & \\
\hline $\mathrm{R}^{2}$ & & & $7,9 \%$ & $7,0 \%$ & & & \\
\hline
\end{tabular}

N1: número incluido en bivariado.

P1: significancia bivariada (regresión logística ponderada)

P2: significancia ajustada por regresión logística múltiple ponderada, modelo inicial

P3: significancia ajustada por regresión logística múltiple ponderada, modelo final

B3: coeficiente, regresión logística múltiple ponderada, modelo final

S3: error estándar, regresión logística múltiple ponderada, modelo final

O3: razón de chances (exp(B3)), regresión logística múltiple ponderada, modelo final

LI: límite inferior de 95\% de confianza para O3 (aproximación normal)

LS: límite superior de 95\% de confianza para O3 (aproximación normal)

$\mathrm{NX}$ : número incluido en regresiones logísticas

$\mathrm{R}^{2}$ : varianza explicada por el modelo (McFadden)

\section{DISCUSIÓN}

Es preciso mencionar que en los niños menores de cinco años, tanto en el caso peso para la talla (P/T) como para el IMC, las $2 z$ son el punto de corte que la OMS denomina sobrepeso ${ }^{(27,28)}$, estimación que corresponde a un percentil mayor que el 95 (1,64z); el cual ha sido llamado obesidad por otras organizaciones incluyendo el Centro de Prevención y Control de Enfermedades (CDC) ${ }^{(29)}$. Para efectos de análisis del presente artículo, usaremos el punto de corte propuesto por la OMS considerando en una sola categoría, de acuerdo con lo empleado con mayor frecuencia en la literatura nacional (la prevalencia nacional usando $3 z$ es $3,4 \%$ en el MONIN), así como por el hecho de que la obesidad es una condición clínica relacionada con el exceso de grasa corporal, por lo que la medición de indicadores antropométricos sería solo una estimación (27).

El presente estudio reporta una prevalencia de sobrepeso y obesidad del $6,9 \%$, lo que implicaría un incremento de casi tres puntos con relación al $4 \%$ observado en el primer estudio nacional durante el año
$1984^{(10) ;}$ incremento en 24 años similar a lo descrito en EUA ${ }^{(30)}$. Sin embargo, estas mediciones usan diferentes criterios para realizar la categorización; en 1984 se usó lo propuesto por el National Center for Health Statistics (NCHS) ${ }^{(31)}$, y como se refirió previamente, en este estudio usamos lo propuesto por la OMS ${ }^{23)}$. Sin embargo, para efectos de comparación se ha calculado la prevalencia MONIN con la referencia del NCHS en un $5,7 \%$; lo que podría considerarse como un incremento de la prevalencia de obesidad y sobrepeso infantiles.

Estudios realizados en Latinoamérica dan a conocer diferentes prevalencias de sobrepeso y obesidad tanto en Argentina (7,3\%), Bolivia (6,5\%), Brasil (4,9\%), Chile (7\%) y Colombia $(2,6 \%)$, con valores cercanos a lo encontrado en el presente estudio, no obstante, la clasificación usada corresponde al $\mathrm{NCHS}{ }^{(32)}$ lo mismo que todos los estudios que se han publicado a nivel nacional (10-11).

La localización del sobrepeso y obesidad se hace más prevalente en los estratos donde se han alcanzado un mayor desarrollo social: Lima metropolitana y resto de la 
costa. Es en esos ámbitos donde existe una mayor densidad poblacional, mejores ingresos económicos, mayores niveles de educación, mejor nivel de urbanización, entre otros, que de cierto modo pueden reflejar los cambios que se vienen dando como expresión de la globalización ${ }^{(33)}$, distribución geográfica que se mantiene para el caso de los adultos ${ }^{(34)}$. Es importante remarcar que en los países en vías de desarrollo donde aún persisten los problemas nutricionales caracterizados por el déficit de energía (desnutrición) y de micronutrientes (anemia nutricional), se viene observando un ambiente obesogénico favorecido por la disminución de la actividad física y el aumento de la disponibilidad de alimentos de alta densidad energética ${ }^{(9)}$.

La distribución de la población estudiada por grupos de edad muestra que los que se encuentran más afectados son los niños de 6 a 11 meses $(13,6 \%)$ y los de 0 a 5 meses (12,1\%); en resumen, en el primer año de vida. Esto podría explicarse por que la mayoría de los niños están aún con lactancia materna, lo que sumado a la alimentación complementaria afectaría el equilibrio energético. Por otro lado, la tendencia en cuanto a las prevalencias en relación a los grupos de edad es similar a un estudio que realizó Tazza ${ }^{(11)}$ en base a los datos de las ENDES de los años 1992, 1996 y 2000.

Por otro lado, hay una mayor proporción de niños con sobrepeso y obesidad en aquellos que tuvieron un peso al nacer superior a 2500 gramos, condición similar a lo descrito en niños españoles ${ }^{(35)}$. Se sugiere que el medio ambiente fetal influiría en el desarrollo de la obesidad; en ese sentido, un estudio llevado a cabo en Israel en 35000 jóvenes reclutados al Ejército, mostró que los nacidos con pesos mayores tenían IMC más altos ${ }^{(36)}$. Adicionalmente, hay considerables evidencias que los niños y adultos obesos que tuvieron bajo peso al nacer son más vulnerables a enfermedades cardiovasculares y a diabetes mellitus tipo $2^{(37)}$.

Si bien existen algunas limitaciones, el MONIN es una aproximación seria para estimar la prevalencia de sobrepeso y obesidad, puesto que parte de una encuesta de base poblacional con un muestreo probabilístico representativo a nivel nacional y a los cinco estratos macro ya mencionados; además, se ha distribuido aleatoriamente en el tiempo. Sus limitaciones provienen de las pérdidas (conglomerados $8 \%$, hogares $13 \%$, antropometría $4 \%$ ) y de su precisión (reflejada en los márgenes de confianza 2 a $4 \%$ nacional y por estratos); lo cual se ha contrarrestado con la calibración correspondiente de los instrumentos de medición y del personal encargado.

En conclusión, el sobrepeso y obesidad en niños menores de cinco años, constituye un problema importante en materia de salud pública, y es más frecuente en aquellos que viven en Lima Metropolitana, que están en el primer año de vida o que tienen un peso al nacer superior a 2 500 gramos.

Se recomienda que la prevención del sobrepeso y la obesidad debe ser una estrategia prioritaria de salud, cuyo abordaje debe de iniciarse precozmente desde la infancia, este debe de ser multidisciplinario e incluir a la familia y escuela (microambiente), así como, autoridades locales, medios de comunicación, universidades, empresarios, entre otros (macro-ambiente).

\section{Contribuciones de Autoría}

JPR participó en la concepción y redacción del artículo; MMC participó en la concepción, análisis y redacción del artículo, así como en el diseño y la ejecución de MONIN; MCS participó en la concepción, análisis, interpretación, redacción del artículo así como en el diseño y la ejecución de MONIN y JSA participó en la concepción y ejecución de MONIN.

\section{Fuentes de Financiamiento}

El MONIN fue financiado por el Centro Nacional de Alimentanción y Nutrición (CENAN), Instituto Nacional de Salud, Lima, Perú.

\section{Conflictos de Interés}

Los autores declaran no tener conflictos de interés.

\section{REFERENCIAS BIBLIOGRÁFICAS}

1. World Health Organization. Preventing and managing the global epidemic. Report of a WHO Consultation on Obesity. World Health Organ Tech Rep Ser. 2000;894:(i-xii):1-253.

2. Sorensen $\mathbf{T}$. The changing lifestyle in the world. Body weight and what else?. Diabetes Care. 2000;23(1):1-4.

3. Ceschi M, Gutzwiller F, Moch H, Eichholzer M. Epidemiology and pathophysiology of obesity is a cause of cancer. Swiss Med Wkly. 2007;137(3-4):50-6.

4. Berenson G, Srinivas S, Bao W, Newman W, Tracy R, Wattigney W. Association between multiple cardiovascular risk factors and atherosclerosis in children and young adults. N Engl J Med. 1998;338(23):1650-6.

5. Ogden C, Carroli M, Curtin L, McDowell M, Tabak C, Flegal K. Prevalence of overweight and obesity in the United States 1999-2004. JAMA. 2006;295(13):154955.

6. Broyles S, Katzmarzyk P, Srinivasan S, Chen W, Bouchard C, Freedman D, et al. The pediatric obesity epidemic continues unabated in Bogalusa, Luisiana. Pediatrics. 2010;125(5):900-5.

7. Branca $\mathbf{F}$, Nikogosian $\mathbf{H}$, Lobstein $\mathbf{T}$. The challenge of obesity in the WHO European Region and the strategies for response. Geneva: World Health Organization; 2007. 
8. James W, Rigby N, Leach R, Kumanyika S, Lobstein T, Swinburne B. Global strategies to prevent childhood obesity: forging a societal plan that works. London: International Association for the Study of Obesity; 2006.

9. Schefer Elinder L, Jansson M. Obesogenic enviromentsaspects on measurement and indicators. Public Health Nutrition. 2008;12(3):307-15.

10. Ministerio de Salud de Perú, Instituto Nacional de Estadistica. Encuesta Nacional de Nutrición y Salud (ENNSA 1984). Lima: INE; 1986.

11. Tazza R, Bullón L. Obesidad o desnutrición?. Problema actual de los niños peruanos menores de 5 años. An Fac Med. 2006;67(3):214-23.

12. Instituto Nacional de Salud, Centro Nacional de Alimentación y Nutrición. Monitoreo Nacional de Indicadores Nutricionales (MONIN) 2003. Lima: INS; 2003 [citado el 25 de abril 2011]. Disponible en: http://www.ins. gob.pe/insvirtual/BiblioDig/MONIN/M2003/ITM2003.pdf

13. Instituto Nacional de Salud, Centro Nacional de Alimentación y Nutrición. Monitoreo Nacional de Indicadores Nutricionales (MONIN) 2004. Lima: INS; 2004 Disponible en http://www.ins.gob.pe/insvirtual/BiblioDig/ MONIN/M2003/ITM2004.pdf.

14. Pajuelo J, Bernui I, Rocca J, Torres L, Soto L. Marcadores bioquìmicos de riesgo cardiovascular en una población adolescente femenina con sobrepeso y obesidad. An Fac Med. 2009;70(1)7-10.

15. Pajuelo J, Rocca J, Gamarra M. Obesidad infantil: sus características antropométricas y bioquímicas. An Fac Med. 2003;64(1):23-9.

16. Pajuelo J, Bernui I, Nolberto V, Peña A, Zevillano L. Sindrome Metabólico en adolescentes con sobrepeso y obesidad. An Fac Med. 2007;68(2):143-9.

17. Mahoney L, Burns T, Stanford W, Thompson B, Witt D, Rost C, et al. Coronary risk factors measured in childhood and youg adults life are associated with coronary artery calcification in young adults: the Muscatine Study. J Am Coll Cardiol. 1996;27(2):277-84.

18. Zhu W, Huang X, He J, Li M. Arterial intima media thickening and endothelial dysfunction in obese children. Eu J Pediatr. 2005; 164(6):337-44

19. Puhl M, Heuer $\mathbf{C h}$. The Stigma of Obesity: a review and update. Obesity. 2009;17(5):941-60.

20. Instituto Nacional de Salud, Centro Nacional de Alimentación y Nutrición. Monitoreo Nacional de Indicadores Nutricionales (MONIN). Lima: INS; 2008. Disponible en http://www.ins.gob.pe/portal/jerarquia/5/311/ monitoreo-nacional-de-indicadores-nutricionales-monin/ jer.311.

21. Campos M, Ricaldi R, Miranda M; Equipo MONIN. Diseño del Monitoreo Nacional de Indicadores Nutricionales (MONIN) Perú 2007-2010. Rev Peru Exp Salud Publica. 2011;28(2):210-21.

22. Instituto Nacional de Salud, Centro Nacional de Alimentación y Nutrición. La Medición de la Talla y e Peso. Guia para el personal de salud del primer nivel de atención. Lima: INS; 2004.

23. Organización Mundial de la Salud. Patrones de Crecimiento Infantil de la OMS. Geneva: Departamento de Nutrición para la Salud y el Desarrollo-OMS; 2006.
Disponible en http://www.who.int/childgrowth/es/

24. Pajuelo J, Medrano M. El uso de diferentes poblaciones referenciales en el diagnóstico de los principales problemas nutricionales en niños y adolescentes. An Fac Med. 2009;70(3):193-8.

25. Díaz J. Nuevo Mapa de Pobreza del Fondo de Cooperación para el Desarrollo Social (FONCODES) 2006. Lima: FONCODES; 2006.

26. Statistical Package for Social Science. SPSS Complex Samples 15.0. Chicago, IL: SPSS; 2006.

27. World Health Organization. Physical status: the use and interpretation of anthropometry. Report of a WHO Expert Committee. Tehcnical Report Series 854. Geneva: WHO; 1995.

28. World Health Organization. Training course on child growth assessment. Module C: interpreting growth indicators. Geneva: WHO; 2008.

29. Himes $\mathbf{J H}$. Challenges of accurately measuring and using $\mathrm{BMI}$ and other indicators of obesity in children. Pediatrics. 2009;124 (Suppl 1):S3-22.

30. Kim J, Peterson K, Scalon K, Fitzmaurice S, Must A, Oken E, et al. Trends in overweight from 1980 through 2001 among preschool aged children enrolled in a Health Maintenance Organization. Obesity. 2006;14(7):110712.

31. Organizaciòn Mundial de la Salud (OMS). Medición del cambio del estado nutricional. Directrices para evaluar el efecto nutricional de programas de alimentación suplementaria destinados a grupos vulnerables. Ginebra: OMS; 1983

32. De Onis $\mathbf{M}$, Blossner $\mathbf{M}$. Prevalence and trends of overweight among preschool children in developing countries. Am J Clin Nutr. 2000;72(4):1032-9.

33. Huynen M, Vallebreg L, Martens P, Benavides B. The epidemiological Transition in Peru. Pan Am J Public Health. 2005;17(1):53-9.

34. Instituto Nacional de Salud, Centro Nacional de Alimentación y Nutrición. Encuesta Nacional de Indicadores Nutricionales, Bioquímicos, Socioeconómicos y Culturales relacionados con las enfermedades crónicas degenerativas. Lima: INS; 2006.

35. Aranceta-Bartrina J, Perez-Rodrigo C, Ribas-Barba L, Serra-Majern L. Epidemiología y factores determinantes de la obesidad infantil y juvenil en España. Rev Pediatr Aten Primaria. 2005;7(Supl 1):S13-20.

36. Seidman D, Laor A, Gale R, Stevenson D, Danon Y. A longitudinal study of birth weight and being overweight in late adolescence. Am J Dis Child. 1991;145(7):782-5.

37. Barker D, Eriksson J, Forber T, Osmond C. Fetal origins of adult disease strength of effects and biological bias. Int $\mathrm{J}$ Epidemiol. 2002;31(6):1235-9.
Correspondencia: Marianella Miranda Cuadros

Dirección: Tizón y Bueno 276, Lima 11, Perú.

Teléfono: (511) 617-6200 Anexo: 6605

Correo electrónico: mymirandac@gmail.com 